\title{
Structural Analysis and Optimization of Pitman Arm
}

\author{
Sujit P. Narwade, Balaji D. Kshirsagar, Subim N. Khan, Kunal M. Nawasagare \\ Department of Mechanical Engineering, JSPM'S RSCOE, SPPU, PUNE
}

(Received 11 October 2020; Accepted 7 July 2021)

DOI: https://doi.org/10.36224/ijes.140205

\begin{abstract}
Now a day's increases in vehicle demand at a lower rate are necessity of vehicle manufacture. Expectation in terms of durability, efficiency and cost is raising exponentially by customers. Over designed components in vehicle leads to increase weight and reduced efficiency. Pitman arm used to steer vehicle can be redesigned and modified for achieving less weight and thus cost. Existing pitman arm component available in market will be reverse engineered and CAD modeled using CATIAV5software.Finiteelement Discretization \& Analysis will be carried out using Ansys package. FEA will help in finding out high strain locations in component and also defining area which can be modified. Strain gauging will be done on areas of high strain location indicated by FEA software. Fixture will be designed for mounting component on UTM. Machining will be done to remove excess material from component. Comparative analysis will be done between experimental and FEA results. Conclusion and future scope will be suggested.
\end{abstract}

Keywords: Pitman Arm, Optimization, CATIA, FEA

\section{Introduction}

The Pitman arm is an important art of steering component in an automobile or truck. The arm is supported by the sector shaft and supports the drag link or center link with a ball joint. It transmits the motion it receives from the steering box into the drag (or center) link, causing it to move left or right to turn the wheels in the appropriate direction. The idler arm is attached between the opposite side of the center link from the Pitman arm and the vehicle's frame to hold the center or drag link at the proper height. A worn ball joint can cause play in the steering, and may get worse over time. Rigid bodies have wide ranging applications across fields including molecular dynamics, robotics, machine assembly, human motion, and computer graphics for video games and feature films. In many applications, the focus is on kinematics or inverse kinematics, and dynamic behavior is of secondary or limited interest with only a few instances of predictable contact and collision, e.g. consider manipulating a robotic arm in a controlled environment. Multibody simulation consists of analysing the dynamic behaviour of a system of interconnected bodies comprised of flexible and/orrigidcomponents. Thebodiesmaybeconstrainedwithrespecttoeachothervia a kinematically admissible set of constraints modelled as joints. These systems can represent an automobile, an aircraft as an assemblage of rigid and flexible parts, a robot with manipulator arms, and soon. In all such cases, the components may undergo large rotation, large displacements.

The pitman arm is also called steering arm, it is a linkage which is attached at one side to the steering box (through sector shaft) at the bottom of the steering wheel shaft and on the other side to the track rod which is attached at the other end to the idler arm. When the steering wheel process is turned left operation side or right operation side, a worm gear at the bottom of the steering shaft turns a set of teeth. That action moves a gear that activates the pitman arm, causing the steering linkage to move the 
wheels. The steering arm system is part of an older recirculating ball steering system which is still used primarily in some full-size SUVs and trucks as compared with the smoother- handling rack and pinion steering mechanism more commonly used in automobiles.

A properly functioning pitman arm process system, 1) precisely directs the movement process of all the other steering links system, 2) limits wheel wobble on bumpy surfaces, 3) assures full wheel turning radius and 4 ) helps to reduce steering wheel vibration.

\section{Literature review}

1.Deping Wang et al. [1] The planetary gear set and steering gear are established kinematic models of, based on the analysis of the transmission mechanism of angle superposition with Active Front Steering system (AFS). A controller of different verity steering ratio for Active Front Steering system is designed, and virtual road tests are made in Car Maker driver vehicle-road simulation environment. The results of simulation tests validate the controller performance and the advantage of variable steering ratio function, also show that the driving comfort is improved at low speed especially, due to the Active Front Steering system alters the steering ratio according to the driving situation.

2.Liping Chen et al. [2] A optimization procedure for the optimal synthesis of a double-axle steering mechanism used in truck with dynamic loads is presented. A multimode model of double-axle steering mechanism is presented to characterize the leaf spring effect. The domination of dynamic loads, the motion interference of steering linkage resulted from the elastic deformation of leaf spring, and the effects of wheel slip angles and the position discrepancies of wheel speed rotation centers are studied systematically. An optimization method based on target cascading methodology is proposed to classify the design variables of double-axle steering mechanism into four levels. A double-axle steering mechanism for a heavy-duty truck is utilized to demonstrate the validity of the proposed method. The simulation results indicate that the hierarchical optimization procedure is effective and robust. And as a result, it will surely be widely used in engineering.

3.Prof. S.S Kelkar et al. [3] Steering system is used to steer the front wheels in response to driver inputs in order to provide overall directional control of the vehicle. Thus, Steering system plays very important role in vehicle handling characteristics. Pitman arm plays a very important role in steering system as it transmits the steering movement to the wheel. The Pitman arm is a linkage attached to the sector shaft of the steering box and track rod that converts the angular motion of the sector shaft into the linear motion needed to steer the wheels. The Pitman arm is supported by the sector shaft and supports the drag link or center link with a ball joint. It transmits the motion it receives from the steering box into the drag (or center) link, causing it to move left or right to turn the wheels in the appropriate direction. Performance study is carried out followed by static structural analysis and optimization to minimize the weight of the pitman arm and thereby reducing the material cost. Optimized model is then verified by physical testing.

4.Cristina Elena Popa et al. [4] The dissertation documents the design project for the steering system and suspension of the 2005 Formula SAE-A racer car made at the University of Southern Queensland. The dissertation includes a review of current automotive steering and suspension systems followed by the review of Formula SAE-A restrictions and design requirements. A thorough analysis of 2004 USQ racer car has been included in order to establish the areas of design modifications followed by the actual design with all the technical specifications required.

5.S.P. Deshmukh et al. [5] Project work present rigid multi body dynamic analysis approach in design. The application of this methodology simplify design process and give correct result. For the case study here work of design done on Ackerman steering mechanism for tipper. In this first according to 
Ackerman conditions basic geometry is design and then optimize it for static loading, modal analysis and then for dynamic forces generated on steering linkages while turning using Rigid Dynamics tool in Ansys. The results obtained from rigid body dynamic analysis are used for testing individual components and if necessary, corrective actions are taken in design in order to sustain that load. Results shows rigid dynamics approach for design reduces time for optimization, simulation and provide the chance to take most corrective action. Rigid dynamics approach is used in modern design techniques for various domains.

\section{Objectives}

Following are the objectives of this project:

- To study and perform static analysis system process on pitman arm under steering load.

- To propose an optimized model which will have better or same performance and reduced weight.

- CAD modelling of Pitman Arm.

- Analysis of Pitman Arm.

- Experimental Testing on Pitman Arm.

- Comparative Analysis between Experimental \& Analysis results.

- Conclusion \& Future scope.

\section{Problem definition}

Now a day's automotive OEM are concentrating on vehicles efficiency and cost effectiveness to reach out with competitors. Pitman arm seems to be an over designed part. Hence to achieve cost effectiveness optimization of less critical components is necessary. Thus concluding that the earlier methods are not suitable for automatic lubrication system there is a need to solve this problem by different mechanism.

\section{Static Analysis}

\subsection{CATIA}

Computer-aided design (CAD) is the use of computer systems (or workstations) to aid in the creation,modification, analysis, oroptimizationofadesign.CADsoftwareisusedtoincrease the productivity of the designer, improve the quality of design, improve communications through documentation, and to create database for manufacturing. CAD output is often in the form of electronic files for print, machining, or other manufacturing operations. The term CADD (for Computer Aided Design and Drafting) is also used.

Its use in designing electronic systems is known as electronic design automation (EDA). In mechanical design it is known as mechanical design automation (MDA) or computer-aided drafting (CAD), which includes the process of creating a technical drawing with the use of computer software.

CAD software for mechanical design uses either vector-based graphics to depict the objects of traditional drafting, or may also produce raster graphics showing the overall appearance of designed objects. However, it involves more than just shapes. As in the manual drafting of technical and engineering drawings, the output of CAD must convey information, such as materials, processes dimensions, and tolerances, according to application-specific conventions.

CAD may be used to design curves and figures in two- dimensional (2D) space; or curves, 
surfaces, and solids in three-dimensional (3D) space.

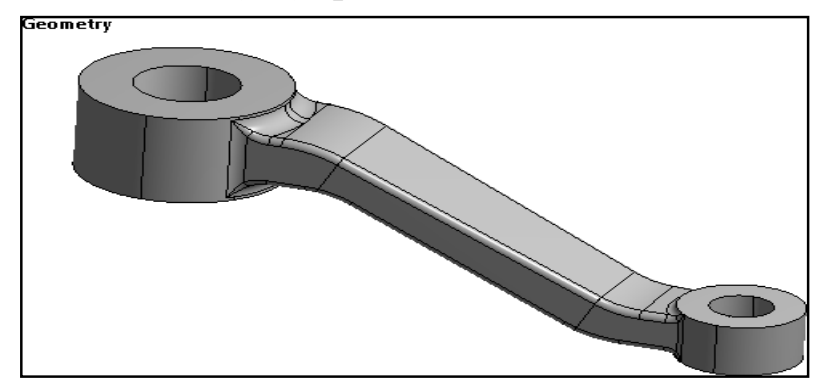

Figure 1: Original CATIA Model Analysis

\subsection{Mesh}

ANSYS Meshing is a general-purpose, intelligent, automated high-performance product. It produces the most appropriate mesh for accurate, efficient Multiphysics solutions. A mesh well suited for a specific analysis can be generated with a single mouse click for all parts in amodel.Fullcontrolsovertheoptionsusedtogeneratethemeshareavailablefortheexpert user who wants to fine-tune it. The power of parallel processing is automatically used to reduce the time you have to wait for meshgeneration.

Creating the most appropriate mesh is the foundation of engineering simulations. ANSYS Meshing is aware of the type of solutions that will be used in the project and has the appropriate criteria to create the best suited mesh. ANSYS Meshing is automatically integrated with each solver within the ANSYS Workbench environment. For a quick analysis or for then ewandin frequent user, ausable mesh can be created with one click of the mouse. ANSYS Meshing chooses the most appropriate options based on the analysis type and the geometry of the model. Especially convenient is the ability of ANSYS Meshing to automatically take advantage of the available core sin the computer to use parallel processing and thus significantly reduce the time to create a mesh. Parallel meshing available without any additional cost or license requirements.

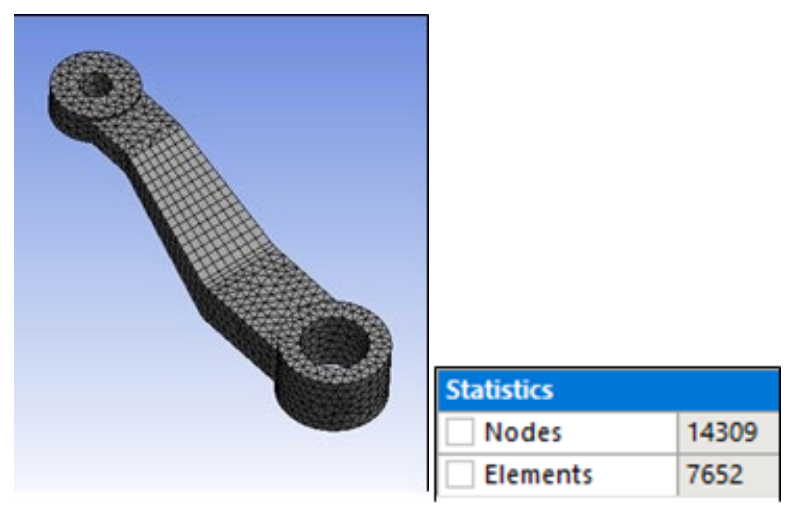

Figure 2: meshing of original model

Total weight of vehicle $(\mathrm{kerb}$ weight + occupied weight $)=930 \mathrm{~kg}$ Weight acting on front right wheel $=930 / 4=232.5 \mathrm{~kg}$

$\mathrm{F}=\mu \mathrm{R}$

Force required to steer vehicle $=0.7 \times 232.5 \times 9.81=1596 \mathrm{~N}$ 


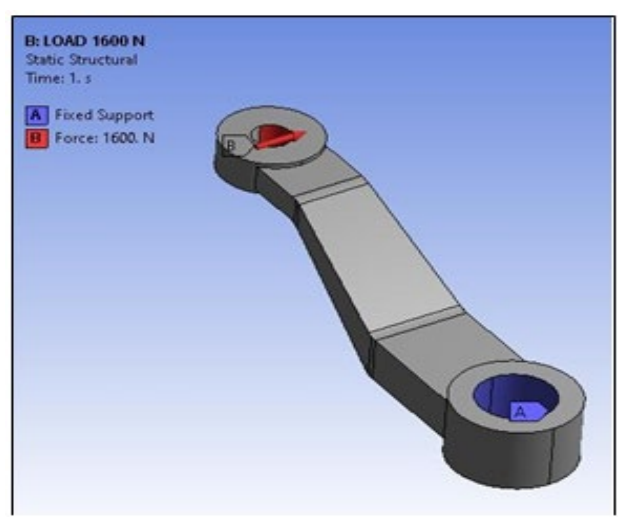

Figure 3: Boundary Condition of Original

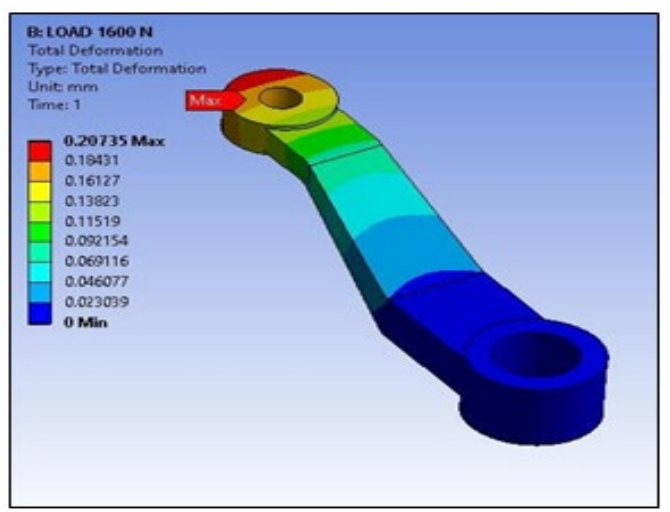

Figure 4: Total Deformation of original model Model

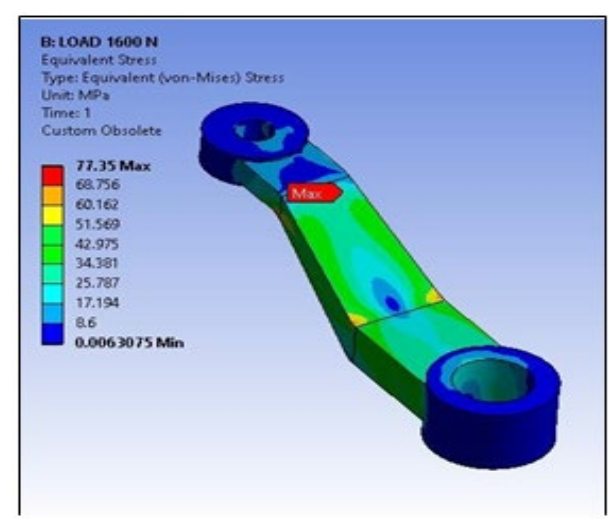

Figure 5: Equivalent stress of original model

\subsection{Topology Optimization}

Topology optimization is a mathematical approach that optimizes material layout within a given design space, for a given set of loads and boundary conditions such that the resulting layout meets a prescribed set of performance targets.
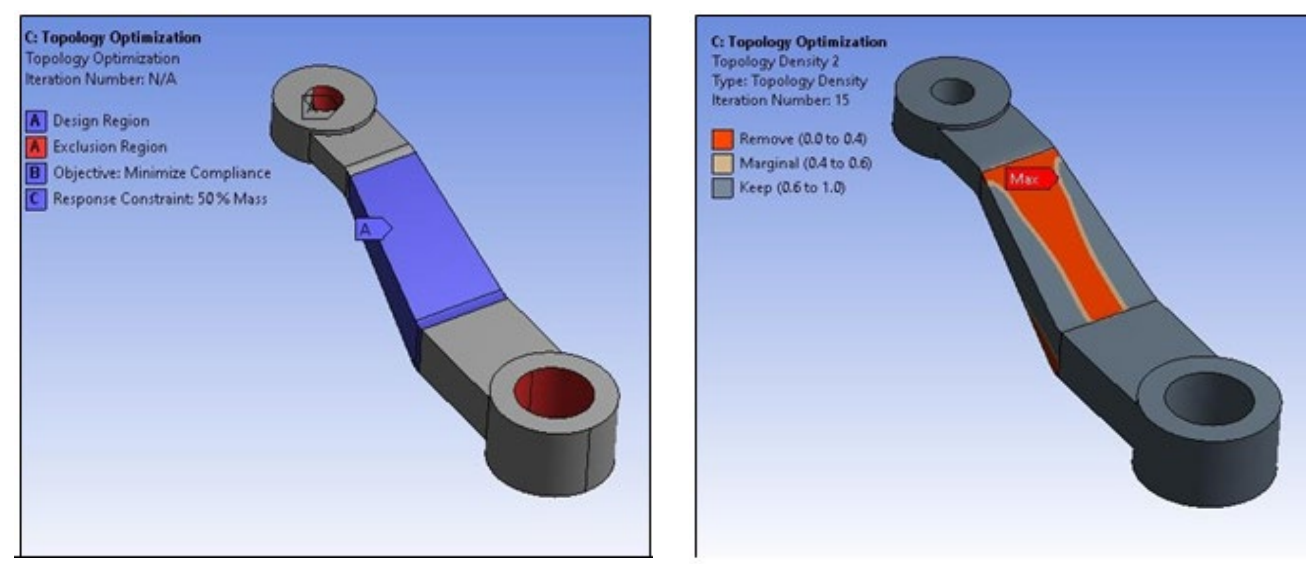

\begin{tabular}{||l|l|}
\hline \hline Original Mass & $1.0207 \mathrm{~kg}$ \\
\hline \hline Final Mass & $0.88633 \mathrm{~kg}$ \\
\hline
\end{tabular}

Figure 6: Topology Optimization 


\subsection{Optimized design}

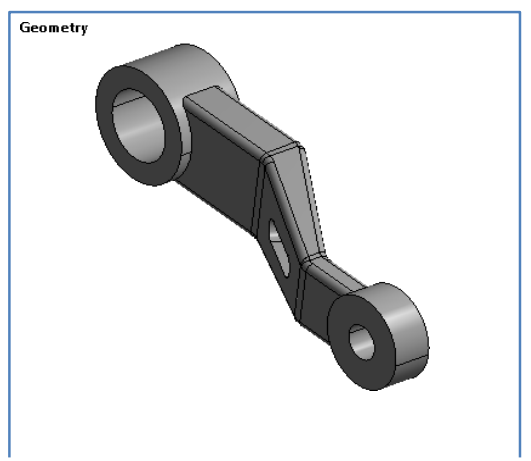

Figure 7: Optimized model
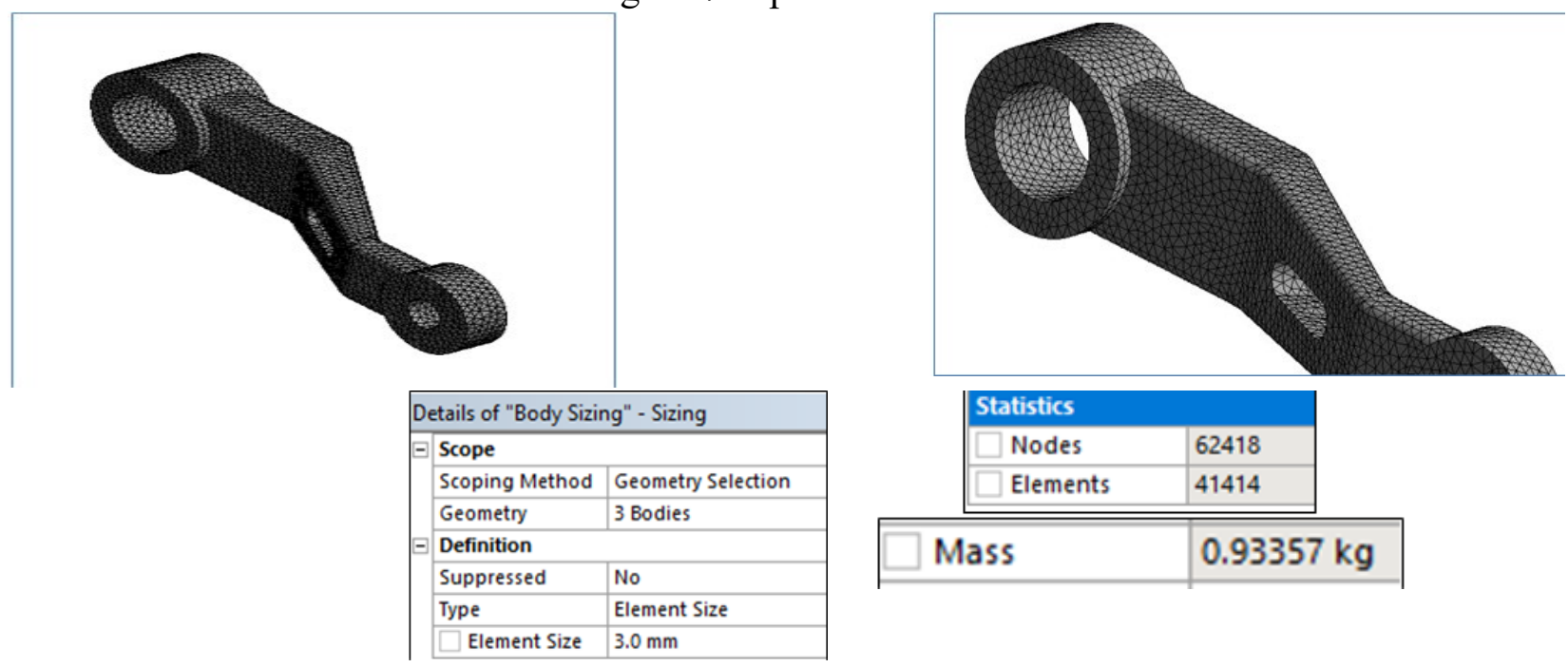

Figure 8: Meshing details

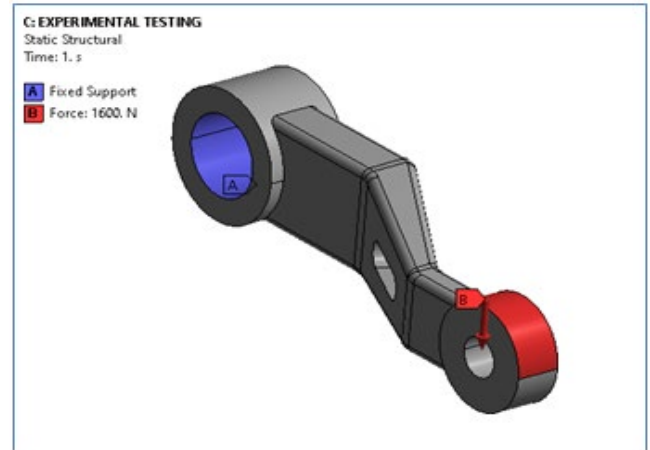

Figure 9: Boundary condition for optimized model

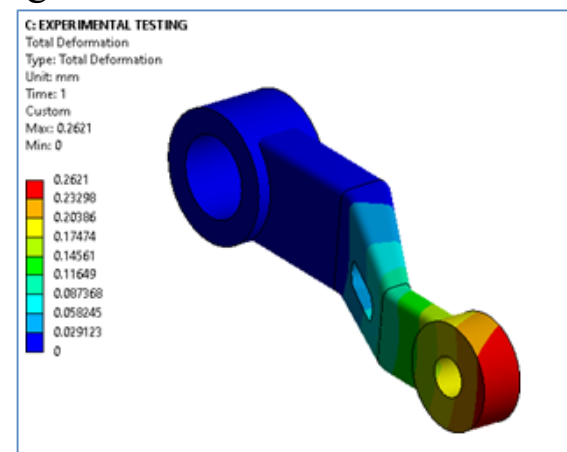

Figure 10: Total deformation for optimized model 


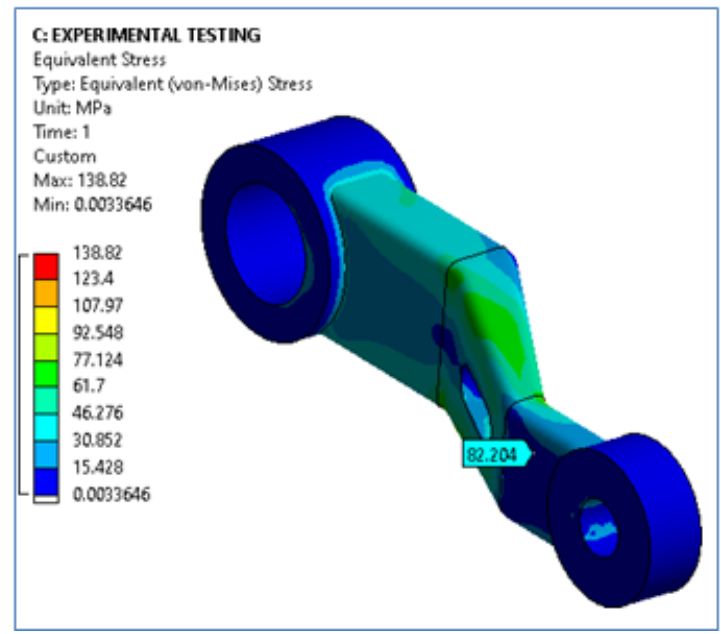

Figure 11: Equivalent stress

\section{Experimental Setup}

A universal testing machine (UTM), also known as a universal tester, materials testing machine or materials test frame, is used to test the tensile strength and compressive strength of materials. An earlier name for a tensile testing machine is a tonometer. The "universal" part of the name reflects that it canperform many standard tensile and compression tests on materials, components, and structures (in other words, that it is versatile). The set-up and usage are detailed in a test method, often published by a standards organization. This specifies the sample preparation, fixturing, gauge length (the length which is under study or observation), analysis, etc. The specimen is placed in the machine between the grips and an extensometer if required can automatically record the change in gauge length during the test. If an extensor meterisnot fitted, thema chine itself canrecordthe displacement between ts cross heads on which the specimen is held. However, this method not only records the change in length of the specimen but also all other extending / elastic components of the testing machine and its drive systems including any slipping of the specimen in the grips. Once the machine is started it begins to apply an increasing load on specimen. Throughout the tests the control system and it sassociated softwareecord theloadand extension or compression ofthe specimen.

Table 1: Specification of UTM

\begin{tabular}{|l|l|l|}
\hline 1 & Max Capacity & $400 \mathrm{KN}$ \\
\hline 2 & Measuring range & $0-400 \mathrm{KN}$ \\
\hline 3 & Least Count & $0.04 \mathrm{KN}$ \\
\hline 4 & Clearance for Tensile Test & $50-700 \mathrm{~mm}$ \\
\hline 5 & Clearance for Compression Test & $0-700 \mathrm{~mm}$ \\
\hline 6 & Clearance Between column & $500 \mathrm{~mm}$ \\
\hline 7 & Ram stroke & $200 \mathrm{~mm}$ \\
\hline 8 & Power supply & 3 Phase, 440 Volts, 50 cycle.A.C \\
\hline 9 & $\begin{array}{l}\text { Overall dimension of machine } \\
\left(\mathrm{L} * \mathrm{~W}^{*} \mathrm{H}\right)\end{array}$ & $2100 * 800 * 2060$ \\
\hline 10 & Weight & $2300 \mathrm{Kg}$ \\
\hline
\end{tabular}




\section{Experimental Testing FEA}

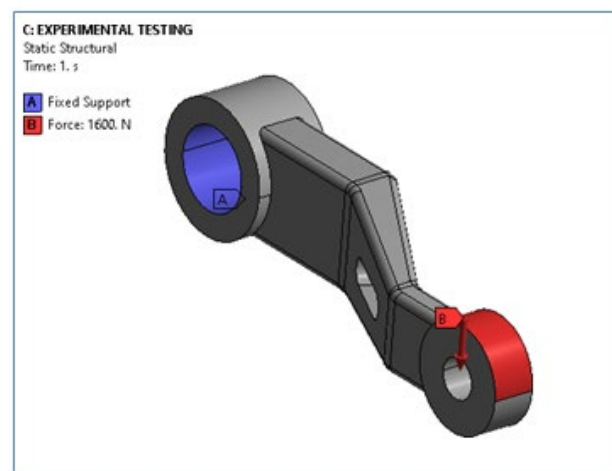

Figure 12: Boundary condition for experimental testing

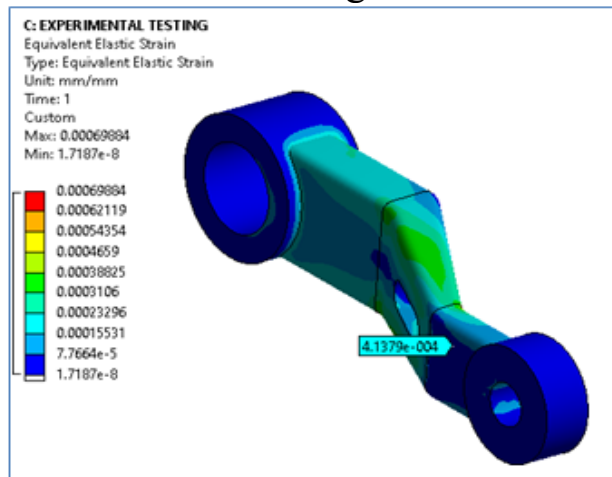

Figure 14: Equivalent elastic strain

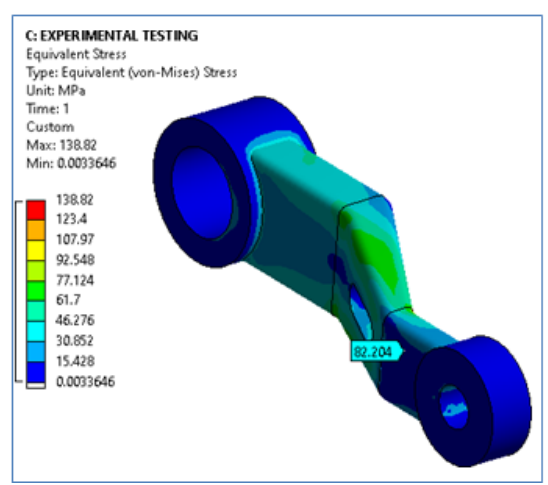

Figure 13: Equivalent stress

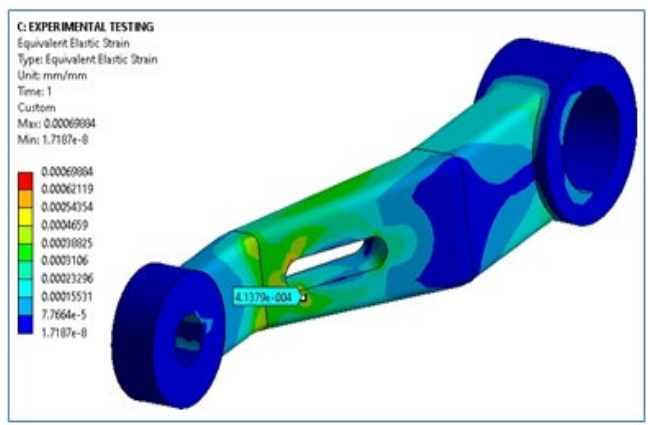

Figure 15: Equivalent elastic strain

\subsection{Experimental procedure}

1) Fixture is manufactured according to component designed.

2) Single force is applied as per FEA analysis and reanalysis is performed to determine strain by numerical and experimental testing.

3) Strain gauge is applied as per FEA results to maximum strained region and during experimental testing force is applied as per numerical analysis to check the strain obtained by numerical and experimental results.

4) Duringstraingageexperimenttwowiresconnectedtostraingageisconnectedtomicro controller through the data acquisition system and DAQ is connected to laptop. Strain gage value are displayed on laptop using DEWESOFTsoftware.

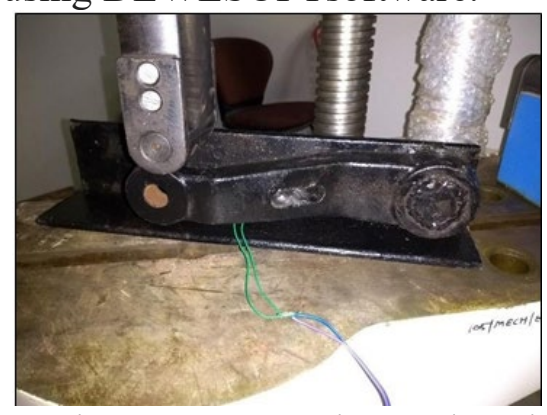

Figure 16: Experimental testing

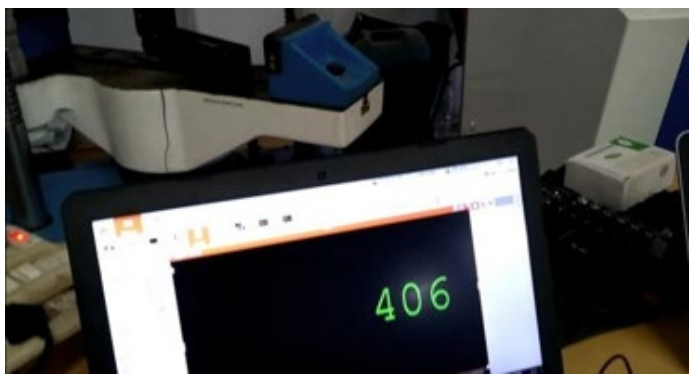

Figure 17: Experimental results 


\section{Conclusion}

In present research pitman arm static and topology optimization analysis is performed to determine stress and deformation. In topology optimization red region is observed so it predicts to removal of material from that area. Static structural analysis of pitman arms is performed to determine deformation and equivalent stress. It is observed that around maximum deformation is $0.20 \mathrm{~mm}$ and equivalent stress is $77.35 \mathrm{MPa}$. An optimized model is obtained from topology optimization technique. In our case original mass is $1.02 \mathrm{~kg}$ but removal of material is to $0.88633 \mathrm{~kg}$ as per software. But itdependsonustoremoval of material by proper design and reanalysis as per existing conditions to sustain boundary condition. Weight reduction of around $8.5 \%$ is observed along with strain measurement of 413 microns and 406 microns by numerical and experimental testing respectively.

\section{References}

[1] Zhenhai Gaoa, Jun Wanga, Deping Wang, "shoulder-elbow Dynamic Modeling and Steering PerformanceAnalysisofActiveFrontSteeringSystem",ADissertationpresentedtothejilinuniversity ofchina

[2] Gang Qin, Ying Sun, Yunqing Zhang and Liping Chen, "Analysis and Optimization of the Double- Axle Steering Mechanism with Dynamic Loads", Open mechanical engineering journal Volume:06 Issue:01 March2012

[3] Sijith PM, Prof. Shashank Gawade, Prof. S.S Kelkar, "CAE Analysis and Structural Optimization of Pitman Arm",University of pune, conference paper,2014

[4] Cristina Elena Popa, "Steering System and Suspension Design for 2005 Formula SAE-A Racer Car", A Dissertation presented to the university of SouthernQueensland,2005

[5] WangChunYana,ZhangYuQia,ZhaoWanZhong,"Multi-objectiveoptimizationofasteeringsystem considering steering modality", Advances in engineering software.126Issue:-09Dec2018

[6] Dongpil Leea , Kyongsu Yia, Sehyun Changb , Byungrim Leeb , Bongchoon Jang, "Robust steeringassisttorquecontrolofelectric-power-assisted-steeringsystemsfortargetsteeringwheel torque tracking", Mechatronics,volume:49 Issue:01 Feb2018

[7] Susanne Schmidt, Wolfgang Seiberl, Ansgar Schwirtz, "Influence of different configurations on steering precision and steering velocity in automotive context ", BMW AG, Forschungs - und Innovationszentrum, Ergonomie und Komfort, Knorrstraße 147, 80788 Munich, Germany volume:46 Issue 2015

[8] Wanzhong Zhao, Han Zhang, Yijun Li, "Displacement and force coupling control design for automotive active front steering", Nanjing University of Aeronautics and Astronautics china, conference paper,Volume:106 Issue:june2018

[9] Chuan Hu, Yechen Qin, Haotian Cao, Xiaolin Song, Kai Jiang, Jagat Jyoti Rath, Chongfeng We, "Lane keeping of autonomous vehicles based on differential steering with adaptive multivariable super-twisting control", Department of Systems Design Engineering, University of Waterloo, Waterloo, Canada,Volume:126 Issue:15june2019. 